\title{
Multicultural Challenges in Employment of People with Disabilities
}

\section{The multicultural mandate in vocational rehabilitation today}

Welcome to this special issue of the Journal of Vocational Rehabilitation. This special issue focuses on "multicultural challenges in employment of people with disabilities". You will note from our focus the prominence of the concepts of culture and employment. It is difficult to imagine that the importance of these two concepts could ever be greater than they are currently during these dawning years of the new millennium.

As this inaugural decade of the 21 st century draws to a close, employment is a major concern amid what has been termed by some as the worst economic downturn since the depression of 1929. Culture is also increasingly important, especially as it relates to ethnicity when one considers the population increases by group in our current decade, i.e., the ten years between 2000 and 2010 according to United States Census Bureau. During this decade, the European-American population is increasing 7\%, African Americans 14\%, Asian Americans 33\%, and Latino Americans 34\%. These numbers make clear why by the year 2050 more than $50 \%$ of the United States population will be non-European American.

Prior to the beginning of this new century, Section 21 of the Rehabilitation Act Amendments of 1992 provided the legislative impetus for the public vocational rehabilitation system to strive to more effectively serve culturally diverse and traditionally underserved populations. Today, the vocational rehabilitation system in the United States (and perhaps other countries) faces a fundamental mandate to "do more with less", within a context of limited resources concomitant with increas- ing demands for services, and resulting in increased pressure toward accountability. Given this mandate, we must find ways to be effective with every population that receives services in our systems. This is the case because we can no longer afford to deliver ineffective services to anyone because then we would be guilty of wasting precious resources. Fulfilling the mandate to serve all individuals effectively raises the bar, and doing so requires that we know how to work successfully with every individual receiving services within an increasingly diverse landscape.

In this issue, we have assembled a cross-section of some of the important, timely and emerging research discussions that are underway today related to employment of individuals with disabilities from culturally diverse backgrounds. Specifically, we have manuscripts that address: (1) the engagement of ethnically diverse individuals with disabilities in the vocational rehabilitation system, (2) application of the behavioral model of service utilization to predict factors associated with vocational rehabilitation use among Latino men with HIV/AIDS, (3) predicting outcomes among women of color with psychiatric disabilities, (4) evaluation and follow-up of supported employment initiatives in Spain from 1995 to 2008, (5) employment and vocational rehabilitation experiences among Latinos with different patterns of acculturation, and (6) employment outcomes after traumatic brain injury among racial and ethnic minority groups.

In a general sense, employment is an avenue to financial self-sufficiency and a respectable quality of life for all Americans. Similarly, employment is still a fundamental outcome needed among individuals with disabilities that correlates positively with optimal 
adjustment to disability. Most everyone seeks to be selfsufficient and obtain an optimal quality of life, and work continues to be a gateway to such outcomes.

Speaking of outcomes, we need to be more cognizant of our research impact these days. In our current phase of research development in the vocational rehabilitation field, we have solid documentation that disability disparities due to cultural orientation exist. Research to date has done a laudable job pinpointing that there is a problem and describing its nature. However, we need strategies that begin to achieve the research outcome of the elimination of the challenges related to disability disparities. Research must embrace a stronger focus on interventions and solutions that tackle these challenges. Given this, we urge each of you to accept the responsibility to help the field progress from mere documentation and description of the problem of disability disparities toward the research outcome of developing, identifying, and promoting strategies that will begin to effectively address, and eventually ameliorate these challenges.

As change agents in our field, we charge each of you to use the take away lessons from the manuscripts in this special issue and advocate that they begin to influence everyday vocational rehabilitation practice in the spirit of knowledge translation. For we not only have a responsibility to produce the research and knowledge that is so derived from it, but equally important, we have a duty to ensure that what we learn in the name of research positively informs everyday vocational rehabilitation practice. Our role in ensuring that research enhances practice would arguably be more effective if we could embrace a multi-pronged advocacy approach. Such an approach would compel us to target effort in each of the five domains of individual, interpersonal, organizational/institutional, community, and public policy context in McLeroy, Bibeau, Steckler, and Glanz's social-ecological model (1988) to influence comprehensive and sustainable change in vocational rehabilitation practice based on lessons learned in research. After all, helping to enhance the lives of individuals with disabilities is the reason that we engage in the disciplined and rigorous inquiry that we call the research endeavor.

We hope that you will find benefit from this issue of the Journal of Vocational Rehabilitation. We offer our appreciation to you, the readership, and to the editor, Dr. Paul Wehman, for making this discussion possible. Finally, we offer special thanks to the following colleagues who served as guest reviewers for the papers in this issue. Their critique and comments were invaluable.
Dr. Faye Belgrave
Dr. James Bethea
Dr. Kelli Gary
Dr. Njeri Jackson
Dr. Pamela Lewis
Dr. Michelle Meade
Dr. Jan Niemeier
Dr. Myra Owens
Dr. Jenelle Pitt
Dr. Paola Premuda-Conti
Dr. Michael Pyles
Dr. Philip D. Rumrill
Dr. Keith Wilson
Dr. Blue Wooldridge
Dr. Tennyson Wright

Allen N. Lewis, Ph.D., CRC Guest Editor

Associate Professor \& Department Chair Department of Rehabilitation Counseling School of Allied Health Professions Virginia Commonwealth University P.O. Box 980330

Richmond, VA 23298-0330, USA Tel.: +1 804827 0914, Fax: +1 8048281321 E-mail: anlewis@vcu.edu

Juan Carlos Arango-Lasprilla, Ph.D. Guest Editor Associate Professor Department of Physical Medicine and Rehabilitation Rehabilitation Psychology and Neuropsychology School of Medicine Virginia Commonwealth University West Hospital, 3rd Floor Room 3-102 1200 East Broad St., P.O. Box 980542 Richmond, VA 23298, USA Tel.: +1 804828 8797, Fax: +1 8048282378 E-mail: jcarangolasp@vcu.edu 